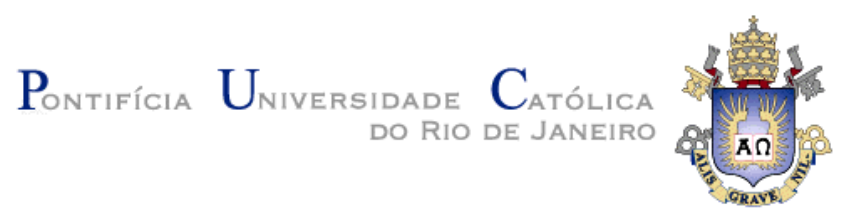

Robson de Oliveira Souza

\title{
Avaliação de Modelos Para a Remoção de Depósitos de Parafina em Dutos Utilizando Pigs
}

Dissertação de Mestrado

Dissertação apresentada como requisito parcial para obtenção do título de Mestre pelo Programa de Pós-Graduação em Engenharia Mecânica da PUC-Rio.

Orientador: Luis Fernando Alzuguir Azevedo

Rio de Janeiro

Abril de 2005 


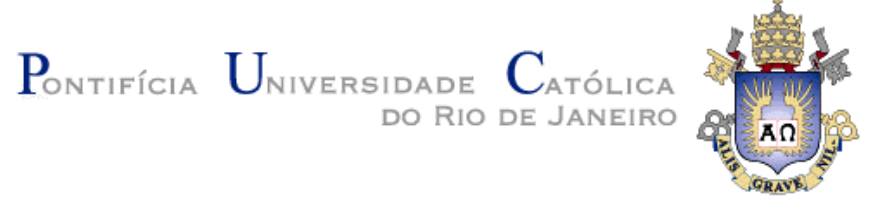

Robson de Oliveira Souza

\begin{abstract}
Avaliação de Modelos para a Remoção de Depósitos de Parafina em Dutos Utilizando Pigs
\end{abstract}

\begin{abstract}
Dissertação apresentada como requisito parcial para obtenção do grau de Mestre pelo Programa de Pósgraduação em Engenharia Mecânica do Departamento de Engenharia Mecânica do Centro Técnico Científico da PUC-Rio. Aprovada pela Comissão Examinadora abaixo assinada.
\end{abstract}

Luiz Fernando Alzuguir Azevedo Orientador

Pontifícia Universidade Católica do Rio de Janeiro

Arthur Martins Barbosa Braga

Pontifícia Universidade Católica do Rio de Janeiro

Paulo Roberto de Souza Mendes

Pontifícia Universidade Católica do Rio de Janeiro

Prof. José Eugenio Leal

Coordenador Setorial do Centro

Técnico Científico - PUC-Rio

Rio de Janeiro, 15 de abril de 2005 
Todos os direitos reservados. É proibida a reprodução total ou parcial do trabalho sem autorização da universidade, do autor e do orientador.

\section{Robson de Oliveira Souza}

Graduou-se em Engenharia Mecânica na Fundação Técnico Educacional Souza Marques em 1992. Especializou-se em Engenharia de Dutos na PUC-Rio em 2001. Trabalha como coordenador de projetos de pesquisa no Centro de Pesquisas e Desenvolvimento da Petrobras (CENPES) no Rio de Janeiro.

Ficha Catalográfica

Souza, Robson de Oliveira

Avaliação de modelos para a remoção de depósitos parafina em dutos utilizando pigs / Robson de Oliveira Souza ; orientador: Luiz Fernando Alzuguir Azevedo. - Rio de Janeiro: PUC-Rio, Departamento de Engenharia Mecânica, 2005.

129 f. ; $30 \mathrm{~cm}$

Dissertação (mestrado) - Pontifícia Universidade Católica do Rio de Janeiro, Departamento de Engenharia Mecânica.

Inclui referências bibliográficas

1. Engenharia mecânica - Teses. 2. Parafina. 3. Depósito. 4. Remoção mecânica. 5. Pig. 6. Garantia de escoamento. I. Azevedo, Luiz Fernando Alzuguir. II. Pontifícia Universidade Católica do Rio de Janeiro. Departamento de Engenharia Mecânica. III. Título. 


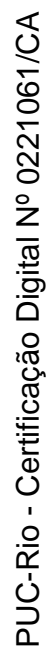

À minha esposa Tamar pelo seu importante apoio durante a elaboração deste trabalho. 
Ao Sr. José Pereira e Sra. Zila, meus pais, pelo amor e cuidado que dispensaram a mim ao longo de minha vida. 
Ao Senhor Deus, criador e sustentador meu, que entre tantas outras bênçãos me concede mais esta importante realização. 


\section{Agradecimentos}

Aos gerentes da Petrobras, Geraldo Spinelli e José Roberto Fagundes Netto por todo o apoio que me deram ao longo do curso de mestrado.

Ao Professor Luís Fernando Azevedo, não apenas pela orientação e participação integral neste trabalho, mas também pela forma amigável e atenciosa com a qual sempre me atendeu.

Ao engenheiro Júlio Manuel Barros Júnior, ao técnico Diogo Felipe Lima da Silva e ao graduando em engenharia mecânica Daniel Prata por todo o apoio que me deram durante a realização dos trabalhos de laboratório.

Aos colegas da Petrobras, engenheiros Divonsir Lopes, Eduardo Ferreira Gaspari, Alberto Gomes de Lima e Antonio Carlos Ferreira Lino pela demonstração de companheirismo através da ajuda que me deram em diversos momentos.

À PUC-Rio e à Petrobras por me permitirem realizar este importante projeto pessoal.

Aos funcionários do Departamento de Engenharia Mecânica da PUC-Rio, pela atenção e boa vontade a mim dispensadas. 


\section{Resumo}

Souza, Robson de Oliveira. Avaliação de Modelos para a Remoção de Depósitos de Parafina em Dutos Utilizando Pigs. Rio de Janeiro, 2005. 129p. Dissertação de Mestrado - Departamento de Engenharia Mecânica, Pontifícia Universidade Católica do Rio de Janeiro.

Pigs ainda são a ferramenta mais utilizada pela indústria para remover depósitos de parafina em linhas submarinas de petróleo. A passagem de Pigs é considerada uma operação de risco, pois existe a possibilidade do gradiente de pressão disponível para deslocar o Pig não ser suficiente para vencer as forças de contato entre o Pig e a parede do tubo e as forças necessárias para remoção do depósito de parafina. Neste trabalho realizou-se um estudo experimental visando o levantamento de dados de laboratório sobre as forças de contato Pig-tubo para alguns tipos de Pigs comumente usados em operações de campo. Além disso, foram também determinadas forças para remoção de depósitos de parafina fabricados no laboratório sob condições controladas. Foi projetada e construída uma seção de testes onde os Pigs ensaiados eram puxados com velocidade constante através de trechos de tubo, sendo a força trativa de puxada monitorada continuamente por células de carga. Os resultados de força de contato Pig-tubo sem depósito de parafina foram comparados com modelos teóricos disponíveis na literatura. Para o caso dos experimentos onde depósitos estavam presentes, as forças necessárias para a quebra dos depósitos foram também comparadas com o único modelo disponível na literatura. Os resultados obtidos mostraram que, para Pigs de disco e de espuma os modelos disponíveis prevêem as forças de contato Pig-tubo com incerteza aceitável. Para o caso de remoção de parafina a comparação com os experimentos desenvolvidos mostrou que o modelo disponível consegue estimar a força inicial para a quebra do depósito dentro de uma faixa de incerteza de $\pm 30 \%$ para os Pigs de disco e de espuma. 


\section{Palavras-chave}

Parafina, Depósito, Remoção Mecânica, Pig, Garantia de Escoamento.

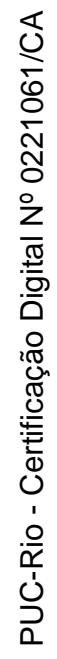




\section{Abstract}

Souza, Robson de Oliveira. Experimental Validation of Models for Predicting the Forces for Removing Wax Deposits in Pipelines Using Pigs. Rio de Janeiro, 2005. 129p. MSc. Dissertation - Departamento de Engenharia Mecânica, Pontifícia Universidade Católica do Rio de Janeiro.

Pigging is still the most widely used method to remove wax deposits in subsea pipelines. The passage of a pig is still considered a risky operation, since the pressure gradient available for driving the pig might not be sufficient to overcome the contact forces between the pig and the wall, as well as the forces required to remove the wax deposit. If this is the case, the pig will be stuck, and costly remediation procedures will have to be implemented. In the present work, an experimental study was carried out aimed at collecting reliable data on the contact forces developed between pigs and the pipe wall for some types of pigs commonly used in field operations. Also, as part of the work, the forces required to remove wax deposits prepared under controlled conditions were recorded. To this end, a test section was specially designed and constructed. In the tests, the pigs were pulled through a set of pipes at constant speed by a winch, while the pulling force was continuously recorded by load cells. The results obtained for the contact forces between disc, foam and conical cup pigs and the pipe wall without wax deposits were compared with models available in the literature. Good level of agreement was obtained. Experimental results obtained for the cases where the test pipes had controlled wax deposits where compared with the only model available in the literature for predicting the forces required to break the wax deposits. The comparisons demonstrated that the measured forces required for breaking the wax deposit can be estimated by the available models within an uncertainty of $\pm 30 \%$. 


\section{Key-Words}

Wax, Deposit, Removal Mechanics, Pig, Flow Assurance.

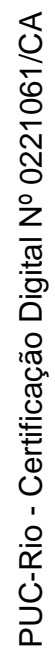




\section{Sumário}

1 Introdução 17

$\begin{array}{ll}1.1 \text { Motivação } & 20\end{array}$

2 Revisão Bibliográfica 23

2.1 Formação de depósitos de parafina 23

2.2 Utilização de pigs para remoção de depósitos de parafina 30

3 Modelos Matemáticos Para Forças de Contato e de Remoção 39

3.1 Modelo para força de contato pig de disco / tubo 39

3.2 Modelo para força de contato pig de espuma / tubo 43

3.3 Modelo para força de remoção de parafina com pig - modo de carga $1 \quad 44$

3.4 Modelo para força de remoção de parafina com pig - modo de carga $2 \quad 47$

4 Descrição do Experimento $\quad 50$

4.1 Seção de testes $\quad 50$

4.1.1 Estrutura de sustentação 51

4.1.2 Tubos de teste de 6 polegadas 53

4.1.3 Guincho elétrico $\quad 55$

4.2 Sistema de aquisição de dados $\quad 57$

4.2.1 Software Labview $\quad 57$

$\begin{array}{ll}\text { 4.2.2 Células de carga } & 60\end{array}$

4.3 Sistema para deposição de parafina 62

$\begin{array}{ll}\text { 4.4 Procedimentos para testes } & 69\end{array}$

4.4.1 Testes para determinação dos casos base $\quad 69$

4.4.2 Testes para remoção de parafina $\quad 71$

5 Resultados dos experimentos para determinação da força de contato pig / tubo casos base 
5.1 Força de contato pig de disco / tubo - caso base 76

5.2 Força de contato pig de espuma / tubo - caso base 80

5.3 Força de contato pig de espuma com raspadores / tubo - caso base 83

5.4 Força de contato pig de copo / tubo - caso base 86

6 Resultados de experimentos para determinação da força de remoção da $\begin{array}{ll}\text { parafina pelo pig } & 90\end{array}$

6.1 Força de remoção de parafina por pig de disco 92

6.2 Força de remoção de parafina por pig de espuma 94

6.3 Força de remoção de parafina por pig de espuma com raspadores 96

6.4 Força de remoção de parafina por pig de copo 98

7 Aplicação de modelos para previsão da força de contato pig / tubo e $\begin{array}{ll}\text { comparaçao com resultados experimentais } & 100\end{array}$

7.1 Modelo para força de contato pig de disco / tubo 102

7.2 Modelo para força de contato pig de espuma / tubo 104

7.3 Modelo para força de contato pig de espuma com raspadores / tubo $\quad 107$

8 Aplicação de modelos para previsão da força de contato pig / parafina e comparação com resultados experimentais 109

8.1 Modelo para força de remoção de parafina - modo de carga 1

8.2 Modelo para força de remoção de parafina - modo de carga 2-pig disco 114

8.3 Modelo para força de remoção de parafina - modo de carga 2 - pig de espuma

8.4 Modelo para força de remoção de parafina - modo de carga 2 - pig de $\begin{array}{ll}\text { espuma com raspadores } & 118\end{array}$

8.5 Modelo para força de remoção de parafina - modo de carga 2-pig copo

8.6 Comparações entre as previsões dos modelos e os resultados experimentais

9 Conclusões 


\section{Lista de figuras}

Figura 1 - Variação da temperatura de solidificação de parafinas normais versus número de átomos de carbono 24

Figura 2 - Parafinas macrocristalinas e microcristalinas $\quad 25$

Figura 3 - Células unitárias dos cristais de parafinas $\quad 26$

Figura 4 - Passagem de pig para remoção de depósitos 30

Figura 5 - Pig de copo 31

Figura 6 - Pig de disco 31

Figura 7 - Pig de espuma $\quad 32$

Figura 8 - Operação para remoção de parafina na Plataforma PXA-3 33

Figura 9 - Aparato experimental de Wang et al (2001) 35

Figura 10 - Forças atuantes no pig 36

Figura 11 - Fases de teste (Wang et al, 2001) 37

Figura 12 - Configuração esquemática do disco modelado (Azevedo, 1997) 40

Figura 13 - Modo de carga 1 (Souza Mendes et al., 1999) 44

Figura 14 - Modo de carga 2 (Souza Mendes et al., 1999) 47

Figura 15 - Desenho esquemático da seção de testes 51

Figura 16 - Estrutura de sustentação 52

Figura 17 - Dimensões finais dos tubos de seis polegadas 53

Figura 18 - Contato entre a chapa de aço e as células de carga 54

Figura 19 - Guincho elétrico 55

Figura 20 - Guincho elétrico com roletes de polipropileno 56

Figura 21 - Sistema de aquisição de dados $\quad 57$

Figura 22 - Painel frontal Labview utilizado nos experimentos 58

Figura 23 - Diagrama de blocos Labview utilizado nos experimentos $\quad 59$

Figura 24 - Células de carga utilizadas nos experimentos 60

Figura 25 - Esquema do circuito elétrico do amplificador 61

Figura 26 - Desenho do dispositivo para deposição de parafina 63

Figura 27 - Dispositivo para deposição de parafina 65

Figura 28 - Retirada do tubo macho com a talha manual 66 
Figura 29 - Retirado do tubo macho com a talha manual 66

Figura 30 - Detalhe da desmontagem do dispositivo para deposição $\quad 67$

$\begin{array}{ll}\text { Figura } 31 \text { - Detalhe do tubo com depósito de parafina } & 67\end{array}$

Figura 32 - Posicionamento do tubo com depósito na seção de teste $\quad 68$

$\begin{array}{ll}\text { Figura } 33 \text { - Posicionamento do pig no aparato experimental } & 73\end{array}$

$\begin{array}{ll}\text { Figura } 34 \text { - Puxada do pig num teste de remoção } & 73\end{array}$

Figura 35 - Saída do pig da seção de teste $\quad 74$

Figura 36 - Desenho esquemático do pig de disco $\quad 76$

Figura 37 - Pig de disco utilizado nos testes $\quad 77$

Figura 38 - Gráfico des testes base com o pig de disco 78

Figura 39 - Gráfico com a média dos testes base com o pig de disco $\quad 78$

Figura 40 - Desenho esquemático do pig espuma RS-7 80

Figura 41 - Pig espuma RS-7 utilizado nos testes $\quad 81$

Figura 42 - Gráfico com a média dos testes base com o pig espuma RS-7 82

Figura 43 - Pig espuma RRR-7 utilizado nos testes 83

Figura 44 - Gráfico com a média dos testes base com o pig espuma com raspadores RRR-7 $\quad 84$

Figura 45 - Desenho esquemático do pig de copo Vantage 86

Figura 46 - Pig de copo Vantage utilizado nos testes $\quad 87$

Figura 47 - Gráfico com a média dos testes base com o pig de copo Vantage 88

Figura 48 - Gráfico comparativo médias dos testes base dos pigs testados 89

Figura 49 - Gráfico dos testes com parafina com o pig de disco 92

Figura 50 - Gráfico com a média dos testes com parafina com o pig de disco 93

Figura 51 - Gráfico com a média dos testes com parafina com o pig de $\begin{array}{ll}\text { espuma RS-7 } & 94\end{array}$

Figura 52 - Gráfico com a média dos testes com parafina com o pig de $\begin{array}{ll}\text { espuma RRR-7 } & 96\end{array}$

Figura 53 - Gráfico com a média dos testes com parafina com o pig de copo Vantage

Figura 54 - Gráfico comparativo das médias dos testes para remoção de parafina 99

Figura 55 - Tela inicial do Simulador PIGSIM 100

Figura 56 - Gráfico Tensão x Deformação da espuma 104

Figura 57 - Gráfico Tensão x Deformação da parafina 110 


\section{Lista de tabelas}

Tabela 1 - Características físicas de algumas parafinas normalmente encontradas no petróleo 23

Tabela $2-\tau_{\max } / \Delta P$ para $\eta=0.3$ (Souza Mendes et al, 1999) 48

Tabela $3-\tau_{\max } / \Delta P$ para $\eta=0.4$ (Souza Mendes et al, 1999) 49

Tabela 4 - Eficiências de remoção na primeira passada de cada pig 91

Tabela 5 - Comparação entre experimentos e modelos - casos base $\quad 121$

Tabela 6 - Comparação entre experimentos e modelos - força de remoção 122 